\title{
Treinamento com Exercício Físico e Doença de Chagas: Função Potencial dos MicroRNAs
}

\author{
Physical Exercise Training and Chagas Disease: Potential Role of MicroRNAs
}

Alex Cleber Improta-Caria ${ }^{1,2}$ e Roque Aras Júnior ${ }^{1}{ }^{(0)}$

Programa de Pós-Graduação em Medicina e Saúde, Faculdade de Medicina, Universidade Federal da Bahia, ${ }^{1}$ Salvador, BA - Brasil

Departamento de Educação Física em Cardiologia do Estado da Bahia, Sociedade Brasileira de Cardiologia, ${ }^{2}$ Salvador, BA - Brasil

\section{Resumo}

A doença de Chagas (DC) é causada pelo Trypanosoma Cruzi. Esse parasita pode infectar vários órgãos do corpo humano, especialmente o coração, causando inflamação, fibrose, arritmias e remodelação cardíaca, e promovendo a cardiomiopatia chagásica crônica (CCC) no longo prazo. Entretanto, poucas evidências científicas elucidaram os mecanismos moleculares que regulam os processos fisiopatológicos nessa doença. Os microRNAs (miRNAs) são reguladores de expressão gênica póstranscricional que modulam a sinalização celular, participando de mecanismos fisiopatológicos da DC, mas o entendimento dos miRNAs nessa doença é limitado. Por outro lado, há muitas evidências científicas demonstrando que o treinamento com exercício físico (TEF) modula a expressão de miRNAs, modificando a sinalização celular em indivíduos saudáveis. Alguns estudos também demonstram que o TEF traz benefícios para indivíduos com DC, porém esses não avaliaram as expressões de miRNA. Dessa forma, não há evidências demonstrando o papel do TEF na expressão dos miRNAs na DC. Portanto, essa revisão teve o objetivo de identificar os miRNAs expressos na DC que poderiam ser modificados pelo TEF.

\section{Introdução}

A doença de Chagas (DC) é uma doença complexa causada pela infecção por Trypanosoma Cruzi (T. Cruzi), um parasita protozoário flagelado, no nível intracelular. ${ }^{1} \mathrm{Na}$ fase aguda, a infecção por T. Cruzi gera grande inflamação dos tecidos e há uma resposta inicial do sistema imune inato na tentativa de combater a parasitemia. ${ }^{2}$

Entretanto, a infecção persiste e o sistema imune adaptativo ativa tanto os linfócitos T, como também as células T citotóxicas e auxiliares, que produzem citocinas, tais como o interferon gama (IFN- $\gamma)$, que podem, por sua vez, levar à morte de parasitas intracelulares ao induzir um aumento nas espécies reativas do oxigênio e nitrogênio, que são microbicidas. Essa

\section{Palavras-chave}

Exercício Físico; Doença de Chagas; MicroRNAs.

Correspondência: Alex Cleber Improta-Caria •

Universidade Federal da Bahia - Programa de Pós-Graduação em Medicina e

Saúde - Rua Dr. Augusto Viana, s/n. CEP 40110-060, Canela, Salvador, BA - Brasil

E-mail: alexcaria.personal@hotmail.com

Artigo recebido em 06/05/2020, revisado em 02/07/2020,

aceito em 16/08/2020

DOI: https://doi.org/10.36660/abc.20200330 infecção também aumenta a expressão do fator de necrose tumoral (TNF- $\alpha$ ), bem como anticorpos específicos para combater o T. Cruzi, que controlam o parasitismo, com o estabelecimento de uma infecção de baixo grau. ${ }^{3}$

Ainda na fase aguda da doença, há um aumento na expressão do peptídeo vasoativo endotelina-1 (ET-1) e da cardiotrofina-1 (CT-1), ambos induzindo a hipertrofia cardíaca, bem como um aumento na expressão da interleucina- 1 beta (IL-1 $\beta$ ), induzindo uma resposta inflamatória e pró-hipertrófica do miocárdio, o que pode iniciar a hipertrofia até mesmo nesse estágio. ${ }^{4,5}$

Com o passar dos anos, a parasitemia diminui; entretanto, os antígenos parasíticos persistem, gerando um infiltrado inflamatório difuso e miocardite, com a presença de linfócitos T CD4+ e CD8+ e macrófagos que continuam a expressar TNF- $\alpha$ e IFN- $\gamma \cdot{ }^{3} \mathrm{O}$ IFN- $\gamma$ tem a função essencial de controlar e combater parasitas, mas também contribui para a patogênese cardíaca, uma vez que lesiona o miocárdio por vários mecanismos moleculares que geram a disfunção miocárdica. ${ }^{6}$

Portanto, a doença evolui e passa para a fase crônica, que pode ser subdividida em duas formas: indeterminada e sintomática. Na forma indeterminada, os indivíduos podem passar anos sem manifestar nenhum tipo de sintoma mais sério, já que existe um equilíbrio entre a parasitemia e o sistema imune do hospedeiro. Entretanto, cerca de 30\% desses pacientes desenvolvem uma forma sintomática ou determinada, que pode desencadear disfunções em vários órgãos, incluindo o coração, desenvolvendo a cardiomiopatia chagásica crônica (CCC) associada à miocardite e a fibrose miofibrilar cardíaca, e, dessa forma, reduzindo a condutividade elétrica cardíaca, levando à remodelação miocárdica. ${ }^{7}$

A CCC gera inflamação do tecido cardíaco, causando miocardite focal ou difusa, hipertrofia ou dilatação do ventrículo esquerdo, e morte progressiva de alguns cardiomiócitos, necrose e depósito de colágeno, ${ }^{8}$ aumentando, assim, o tecido fibrótico, levando à redução de sua capacidade de contração. Esse resultado é geralmente associada a arritmias e insuficiência cardíaca, ${ }^{9}$ mas é possível que os microRNAs (miRNAs) participem desses mecanismos. Em geral, os mecanismos moleculares que regulam esses processos não são bem entendidos.

MiRNAs são pequenos RNAs com comprimento de apenas 18 a 25 nucleotídeos, ${ }^{10}$ proteínas não codificantes, e reguladores da expressão gênica pós-transcricional com a função de inibir ou degradar seus genes alvo. ${ }^{11,12}$ Já se demonstrou que vários tipos de treinamento com exercício físico (TEF) modulam a expressão dos miRNAs. ${ }^{13}$ Entretanto, há poucos artigos na literatura que 
tenham analisado os efeitos do TEF na expressão dos miRNAs na DC. Portanto, o objetivo desta revisão de literatura foi analisar os miRNAs expressos na DC e comparar esse resultado aos miRNAs expressos durante ou depois do TEF.

\section{Doença de Chagas e miRNAs}

Poucos estudos na literatura analisaram o perfil de expressão de miRNAs na DC, seja na fase aguda ou na crônica, bem como a sinalização celular que é regulada pelos miRNAs nessa doença negligenciada. Portanto, este trabalho incluiu todos os estudos que avaliaram o padrão de expressão dos miRNAs na DC (Tabela 1).

\section{Doença de Chagas (fase aguda) e miRNAs}

Durante a fase aguda da DC, os pesquisadores avaliaram a expressão de miRNAs aos 15, 30 e 45 dias após a infecção, e identificaram que os miRNAs se expressaram diferentemente durante a parasitemia e que mudanças no intervalo QT sofreram regulação ascendente: miR-20, miR-20b, miR-21, miR-142, miR-146a, miR-146b, miR-155, miR-182, miR203, miR-222, e descendente: miR-139, miR-145, miR-149, miR-322, miR-503. ${ }^{14}$

Outro estudo realizou uma análise in silico para identificar a expressão diferencial de miRNAs e seus genes alvo em vários processos biológicos durante a fase aguda da infecção pelo T. Cruzi, demonstrando que alguns podem estar associados ao processo patológico, tais como os miRNAs miR-238-3p, miR-149-5p, miR-143-3p, miR-145-5p e miR-486-5p. Outros miRNAs podem estar associados à imunidade e função cardiovascular, por exemplo: miR-10a-5p, miR-16-5p, miR30c-5p, miR-34a-5p, miR-138-5p, miR-146a-5p, miR-149, miR-191-5p, miR-204-5p, miR-320b e miR-653-3p, bem como miRNAs relacionados ao processo de fibrose de tecidos: miR-34a-5p, miR-142-3p, miR-200b-3p e 203a-3p. ${ }^{15}$

\section{Doença de Chagas (fase crônica) e miRNAs}

A expressão dos miRNAs do tecido cardíaco dos pacientes com CCC após o transplante cardíaco foi analisada e comparada à expressão de miRNAs do tecido cardíaco de doadores saudáveis. De todos os miRNAs analisados, cinco tiveram sua expressão reduzida (miR-1, miR-133a, miR- 133b, miR-208a e miR-208b) em pacientes com CCC em comparação ao grupo de controle. ${ }^{16}$ Em contraste, o miR-208a circulante em uma amostra de plasma foi superexpresso em pacientes com DC. Entretanto, eles foram indeterminados na fase crônica. ${ }^{17}$

A superexpressão de miR-19a, miR-21 e miR-29b já foi descrita em pacientes com CCC em comparação a indivíduos saudáveis. Inclusive, na análise histológica do tecido cardíaco de pacientes no estágio final da CCC, identificou-se que, além desses miRNAs mencionados acima, o miR-30a e o miR-199b também são superexpressos na DC. ${ }^{18}$

Esses estudos demonstram que muitos miRNAs participam de vários processos na DC, tanto na fase aguda quanto na crônica. Entretanto, são necessários mais estudos para elucidar o papel desses miRNAs e a sinalização celular que estão regulando na DC, incluindo a importância de terapias e tratamentos que podem modular o padrão de expressão apresentado na doença.

\section{Doença de Chagas e treinamento com exercício físico: miRNAs como possiveis moduladores}

Vários tipos de TEF foram descritos como moduladores da expressão de miRNAs, ${ }^{13}$ em estudos experimentais e clínicos, tais como TEF de natação, ${ }^{20}$ maratona, ${ }^{21}$ corrida em esteira ${ }^{22}$ e treinamento de resistência $(T R)^{23}$ (Tabela 2).

Alguns estudos também relataram a importância do TEF na modulação da expressão dos miRNAs em situações patológicas, bem como em diabéticos, ${ }^{24,25}$ na obesidade, ${ }^{26}$ após o infarto do miocárdio ${ }^{27}$ e com insuficiência cardíaca; ${ }^{22}$ entretanto, o papel do TEF na modulação dos miRNAs na DC ainda não foi evidenciado. A literatura apresenta apenas estudos que demonstraram os efeitos benéficos do TEF na DC; porém eles não analisaram o perfil do miRNA.

Realizando apenas TEF aeróbico com intensidade moderada (50 a 70\% de frequência cardíaca máxima), três vezes por semana, por 30 minutos, em 12 semanas, obteve-se um aumento significativo na capacidade cardiorrespiratória metabólica máxima (VO2), aumento de tempo de exercício, distância percorrida e melhoria de aspectos emocionais, ${ }^{28} \mathrm{e}$, além disso, em associação com um programa de TR, foram obtidos resultados benéficos. ${ }^{29}$

Tabela 1 - MicroRNAs na doença de Chagas

\begin{tabular}{|c|c|c|c|}
\hline MicroRNAs & Fonte & Achados & Referência \\
\hline$\downarrow$ miR-1, miR-133a, miR-133b, miR-208a, miR-208b & Amostras cardíacas & $\begin{array}{c}\text { Associação a distúrbios do tecido conjuntivo } \\
\text { e fibrose }\end{array}$ & 16 \\
\hline$\uparrow \operatorname{miR}-208 b$ & Amostras plasmáticas & $\begin{array}{l}\text { Associação à disfunção cardiovascular e } \\
\text { hipertrofia miocárdica }\end{array}$ & 17 \\
\hline $\begin{array}{l}\uparrow \text { miR-20, miR-20b, miR-21, miR-142, miR-146a, } \\
\text { miR-146b, miR-155, miR-182, miR-203, miR-222 } \\
\downarrow \text { miR-139, miR-145, miR-149, miR-322, miR-503, }\end{array}$ & Amostras cardíacas & $\begin{array}{c}\text { Associação com o intervalo QT (QTC) } \\
\text { corrigido pela frequência cardíaca. } \\
\text { Despolarização e repolarização ventricular. }\end{array}$ & 14 \\
\hline 个 miR-19a, miR-21, miR-29b, miR-30a, miR-199b & $\begin{array}{l}\text { Amostras cardíacas } \\
\text { e modelo celular }\end{array}$ & $\begin{array}{l}\text { Associação à fibrose e remodelação } \\
\text { cardíaca }\end{array}$ & 18 \\
\hline $\begin{array}{l}\uparrow \text { miR-16, miR-26b, miR-190b, miR-3586, let-7f-2 } \\
\downarrow \text { miR-190b }\end{array}$ & $\begin{array}{c}\text { Células } \mathrm{H} 9 \mathrm{c} 2 \text {, infectadas com } \\
\text { T. Cruzi }\end{array}$ & $\begin{array}{l}\text { Associação com crescimento celular, } \\
\text { hipertrofia e sobrevivência celular }\end{array}$ & 19 \\
\hline
\end{tabular}




\section{Artigo de Revisão}

\begin{tabular}{|c|c|c|c|c|}
\hline MicroRNAs & Alvo & Fonte & Tipos de exercícios & Referência \\
\hline \multicolumn{5}{|c|}{ Modelos experimentais in vivo } \\
\hline $\begin{array}{l}\uparrow \text { miR-27a, miR-155 } \\
\downarrow \text { miR-143 }\end{array}$ & ACE, AT1R & Amostras cardíacas & $\begin{array}{l}\text { Ratos Wistar-Kyoto } \\
\text { Treinamento físico na esteira }\end{array}$ & 39 \\
\hline$\uparrow \mathrm{miR}-17-3 p$ & $\begin{array}{l}\text { TIMP-3 } \\
\text { PTEN }\end{array}$ & Amostras cardíacas & $\begin{array}{l}\text { Ratos C57Bl/6 } \\
\text { Modelo de treinamento de nado em rampa } \\
\text { Treinamento em roda voluntário }\end{array}$ & 40 \\
\hline$\uparrow \mathrm{miR}-222$ & HIPK1 & Amostras cardíacas & $\begin{array}{l}\text { Modelo de nado em rampa } \\
\text { Treinamento em roda voluntário }\end{array}$ & 41 \\
\hline $\begin{array}{l}\uparrow \text { miR-19b, miR-30e, miR-133b, miR- } \\
\text { 208a } \\
\downarrow \text { miR-99b, miR-100, miR-191a, miR- } \\
\text { 22, miR-181a }\end{array}$ & $\begin{array}{l}\text { IGF-1 } \\
\text { PI3K/AKT/mTOR } \\
\text { MAPK }\end{array}$ & $\begin{array}{c}\text { Amostras cardíacas } \\
\text { Plasma }\end{array}$ & $\begin{array}{l}\text { Ratos Wistar albinos } \\
\text { Treinamento de natação }\end{array}$ & 42 \\
\hline$\uparrow$ miR-29a, miR-101a & $\begin{array}{l}\text { TG- } \beta \\
\text { fos } \\
\text { COL1A1 }\end{array}$ & Amostras cardíacas & Exercício de corrida intermitente & 43 \\
\hline $\begin{array}{l}\uparrow \text { miR-27a, miR-27b } \\
\downarrow \text { miR-143 }\end{array}$ & $\begin{array}{l}\text { ACE } \\
\text { ACE2 }\end{array}$ & Amostras cardíacas & $\begin{array}{c}\text { Ratos Wistar } \\
\text { Treinamento de natação }\end{array}$ & 44 \\
\hline$\uparrow \mathrm{miR}-126$ & PI3KR2 & $\begin{array}{c}\text { Amostras cardíacas } \\
\text { Plasma }\end{array}$ & $\begin{array}{l}\text { Ratos Zucker } \\
\text { Treinamento de natação }\end{array}$ & 26 \\
\hline$\downarrow$ miR-214 & SERCA2A & Amostras cardíacas & $\begin{array}{l}\text { Ratos Wistar } \\
\text { Treinamento de resistência }\end{array}$ & 23 \\
\hline $\begin{array}{l}\uparrow \text { miR-1 } \\
\downarrow \text { miR-214 }\end{array}$ & $\begin{array}{c}\text { NCX } \\
\text { SERCA2A }\end{array}$ & Amostras cardíacas & $\begin{array}{c}\text { Ratos Wistar } \\
\text { Treinamento de natação }\end{array}$ & 27 \\
\hline $\begin{array}{l}\uparrow \text { miR-29c } \\
\downarrow \text { miR-1, miR-133a, miR133b }\end{array}$ & $\begin{array}{l}\text { COL1A1 } \\
\text { COL3A1 }\end{array}$ & Amostras cardíacas & $\begin{array}{c}\text { Ratos Wistar } \\
\text { Treinamento de natação }\end{array}$ & 45 \\
\hline$\uparrow \operatorname{miR}-126$ & $\begin{array}{l}\text { SPRED1 } \\
\text { PI3KR2 }\end{array}$ & Amostras cardíacas & $\begin{array}{c}\text { Ratos Wistar } \\
\text { Treinamento de natação }\end{array}$ & 46 \\
\hline $\begin{array}{l}\uparrow \text { miR-21, miR-144, miR-145 } \\
\downarrow \text { miR-124 }\end{array}$ & $\begin{array}{l}\text { PTEN } \\
\text { PIK3A } \\
\text { TSC2 }\end{array}$ & Amostras cardíacas & $\begin{array}{c}\text { Ratos Wistar } \\
\text { Treinamento de natação }\end{array}$ & 20 \\
\hline
\end{tabular}

$\uparrow$ miR-336-5p, miR-130b-5p, let7d-3p,

miR-466c-5p, miR-324-3p, miR-

146b-5p, miR-132-3p, miR-21-5p,

miR-187-3p, miR-29b-5p, miR-324-5p,

miR-214-5p, miR-140-5p, miR-152-5p,

miR-99b-5p, miR-130a-5p, miR-455-

5p, miR-27b-3p, miR-23b-3p, miR-

652-5p, miR-199a-3p, miR-223-5p,

miR-421-3p, miR-27a-5p, miR-24-5p,

miR-34a-3p, miR-140-3p, miR-125b-

$5 p$, miR-145a-5p, miR-192-5p, miR-

139-5p, miR-199a-5p, miR-674-3p,

miR-191-5p, miR-28-3p, miR-195-5p,

miR-598, miR-429, miR-224, miR-425,

miR-221

$\downarrow$ miR-701-5p, miR-220, miR-144-3p,

miR-694, miR-485-3p, miR-136-5p,

miR-384-3p, miR-376c-3p, miR-208b-

$3 p$, miR-411-3p, miR-141-5p, miR-

1894-3p, miR-9a, miR-687, miR-451-5p

\begin{tabular}{lccc}
\hline $\begin{array}{l}\uparrow \text { miR-503, miR-465b-5p, miR-542-3p } \\
\downarrow \text { miR-652 }\end{array}$ & & Amostras cardíacas & $\begin{array}{c}\text { Ratos C57BI6 } \\
\text { Treinamento de natação }\end{array}$ \\
\hline & IGF1R & & Ratos Balb/c \\
$\downarrow$ miR-26b, miR-143 & GATA-4 & Amostras cardíacas & Treinamento aeróbico em rodas de metal \\
& NFAT1C & & 47 \\
& GSK3B & & Treinamento de natação \\
$\uparrow$ miR-21, miR-30b & BCL-2 & & \\
$\downarrow$ miR-1 & p53 & Amostras cardíacas & 49 \\
& PDCD4 & &
\end{tabular}

TNF- $a$

COL1A1

MMP9

PTEN

AKT1

AMPK

BCL2
Amostras cardíacas

Ratos Wistar

Treinamento aeróbico de corrida

22 
Artigo de Revisão

\begin{tabular}{|c|c|c|c|c|}
\hline \multicolumn{5}{|l|}{ Continuação } \\
\hline$\uparrow \mathrm{miR}-23 \mathrm{a}, \mathrm{miR}-27 \mathrm{a}$ & $\begin{array}{l}\text { PTEN } \\
\text { Casp7 } \\
\text { Fox01 }\end{array}$ & $\begin{array}{c}\text { Amostras } \\
\text { musculoesqueléticas }\end{array}$ & Exercício de resistência & 50 \\
\hline $\begin{array}{l}\uparrow \operatorname{miR}-29 c \\
\downarrow \text { miR-1 }\end{array}$ & $\begin{array}{l}\text { COL1A1 } \\
\text { COL3A1 }\end{array}$ & Amostras cardíacas & Treinamento de natação & 51 \\
\hline$\uparrow \mathrm{miR}-382$ & & $\begin{array}{l}\text { Amostras de soro, } \\
\text { tecido e células }\end{array}$ & Exercício aeróbico em ratos IR & 25 \\
\hline MicroRNAs & Alvos & Fonte & Tipos de exercícios & Referência \\
\hline \multicolumn{5}{|c|}{ Estudos clínicos } \\
\hline$\uparrow \operatorname{miR}-126, \mathrm{miR}-133$ & CPK & Plasma & $\begin{array}{c}\text { Espiroergometria limitada a um único sintoma } \\
\text { Corrida de maratona } \\
\text { Exercício excêntrico de resistência }\end{array}$ & 52 \\
\hline$\downarrow$ miR-486 & PTEN & Soro & Ciclismo sistemático a 70\% V02max & 53 \\
\hline $\begin{array}{l}\text { 个 miR-1, miR-126, miR-133a, miR-134, } \\
\text { miR-146a, miR-208a, miR-499-5p }\end{array}$ & $\begin{array}{l}\text { CPK } \\
\text { NT-proBNP } \\
\text { hsCRP }\end{array}$ & Plasma & $\begin{array}{l}\text { Corrida de maratona } \\
\text { Imediatamente após a corrida }\end{array}$ & 21 \\
\hline $\begin{array}{l}\text { 个 miR-1, miR-133a, miR-206, miR- } \\
\text { 208b, miR-499 }\end{array}$ & & Plasma & $\begin{array}{l}\text { Corrida de maratona } \\
\text { Imediatamente após a corrida }\end{array}$ & 54 \\
\hline$\uparrow \operatorname{miR}-1, \mathrm{miR}-133 a, \mathrm{miR}-206$ & & Plasma & $\begin{array}{c}\text { Corrida de maratona } \\
\text { Imediatamente após a corrida }\end{array}$ & 55 \\
\hline $\begin{array}{l}\uparrow \text { let-7d-3p, let-7f-3p } \\
\text { miR-29a-3p, miR-34a-5p, miR-125b- } \\
\text { 5pmiR-132-3p, miR-143-3p, } \\
\text { miR-148a-3p, miR-223-3p, miR-223-5p } \\
\text { miR-424-3p, miR-424-5p }\end{array}$ & & Soro & $\begin{array}{l}\text { Corrida de maratona } \\
\text { Imediatamente após a corrida }\end{array}$ & 56 \\
\hline $\begin{array}{l}\uparrow \text { miR-1, miR-30a, miR-133a } \\
\downarrow \text { miR-26a, -29b }\end{array}$ & & Plasma & $\begin{array}{l}\text { Corrida de maratona } \\
\text { Imediatamente após a corrida }\end{array}$ & 57 \\
\hline$\uparrow$ miR-1, miR-133a, miR-206 & & Plasma & $\begin{array}{c}\text { Corrida de maratona } \\
\text { Imediatamente após a corrida }\end{array}$ & 58 \\
\hline $\begin{array}{l}\uparrow \text { miR-1, miR-133a, miR-133b, miR- } \\
\text { 139-5p, miR-143, miR-145, miR-223, } \\
\text { miR-330-3p, miR-338-3p, miR-424 } \\
\downarrow \text { miR-30b, miR-106a, miR-146, } \\
\text { miR-151-3p, miR-151-5p, miR-221, } \\
\text { miR-652, let-7i } \\
\uparrow \text { miR-103, miR-107 } \\
\downarrow \text { miR-21, miR-25, miR-29b, miR-92a, } \\
\text { miR-133a, } \\
\text { miR-148a, miR-148b, miR-185, } \\
\text { miR-342-3p, miR-766, let-7d }\end{array}$ & & Plasma & $\begin{array}{c}\text { Teste cicloergômetro } \\
1-3 \mathrm{~h} \text { após o exercício } \\
\text { Ciclo de resistência sistemática } \\
\text { treinamento ergométrico }\end{array}$ & 59 \\
\hline $\begin{array}{l}\uparrow \text { miR-1, miR-133a, miR-133b, miR- } \\
206 \\
\text { miR-485-5p, miR-509-5p, miR-517a } \\
\text { miR-518f, miR-520f, miR-522, miR- } \\
\text { 553, miR-888 }\end{array}$ & & Plasma & $\begin{array}{l}\text { Treinamento intervalado de alta intensidade } \\
\text { Imediatamente após }\end{array}$ & 60 \\
\hline $\begin{array}{l}\uparrow \mathrm{miR}-181 \mathrm{~b}, \mathrm{miR}-214 \\
\uparrow \mathrm{miR}-1, \mathrm{miR}-133 \mathrm{a}, \mathrm{miR}-133 \mathrm{~b}, \mathrm{miR}- \\
208 \mathrm{~b}\end{array}$ & & Plasma & $\begin{array}{l}\text { Teste de esteira em aclive (concêntrico) } \\
\text { Imediatamente após } \\
\text { Teste de esteira em declive (excêntrico) } \\
\text { 2-6 h após o exercício }\end{array}$ & 61 \\
\hline $\begin{array}{l}\uparrow \mathrm{miR}-149 \\
\downarrow \mathrm{miR}-146 \mathrm{a}, \mathrm{miR}-221\end{array}$ & & Soro & $\begin{array}{l}\text { Exercício de resistência } \\
3 \text { dias após o exercício }\end{array}$ & 62 \\
\hline $\begin{array}{l}\uparrow \mathrm{miR}-1, \mathrm{miR}-133 \mathrm{a}, \mathrm{miR}-133 \mathrm{~b}, \mathrm{miR}- \\
\text { 206, miR-208b, miR-499 }\end{array}$ & & Plasma & $\begin{array}{l}\text { Treinamento de resistência sistemático } \\
36-72 \text { h após o exercício }\end{array}$ & 63 \\
\hline $\begin{array}{l}\uparrow \text { miR-1, miR-133a, miR-133b, miR- } \\
181 a \\
\downarrow \text { miR-9, miR-23a, miR-23b, miR-31 } \\
\uparrow \text { miR-1, miR-29b }\end{array}$ & $\begin{array}{l}\text { HDAC4 } \\
\text { NRF1 }\end{array}$ & $\begin{array}{c}\text { Amostras } \\
\text { musculoesqueléticas }\end{array}$ & Teste cicloergômetro, ciclismo & 64 \\
\hline
\end{tabular}




\section{Artigo de Revisão}

\begin{tabular}{|c|c|c|c|c|}
\hline \multicolumn{5}{|l|}{ Continuação } \\
\hline $\begin{array}{l}\uparrow \text { miR-136, miR-200c, miR-376a, miR- } \\
377 \text {, miR-499b, miR-558 } \\
\downarrow \text { miR-28, miR-30d, miR-204, miR-330, } \\
\text { miR-345, miR-375, miR-449c, miR- } \\
\text { 483, miR-509, miR-520a, miR-548a, } \\
\text { miR-628, miR-653, miR-670, miR-889, } \\
\text { miR-1245a, miR-1270, miR-1280, miR- } \\
\text { 1322, miR-3180 }\end{array}$ & & $\begin{array}{c}\text { Amostras } \\
\text { musculoesqueléticas }\end{array}$ & Treinamento de resistência & 65 \\
\hline $\begin{array}{l}\uparrow \text { miR-451 } \\
\downarrow \text { miR-26a, miR-29a, miR-378 }\end{array}$ & & $\begin{array}{c}\text { Amostras } \\
\text { musculoesqueléticas }\end{array}$ & Exercício de resistência & 66 \\
\hline $\begin{array}{l}\uparrow \text { miR-125a, miR-145, miR-181b, miR- } \\
\text { 193a, miR-197, miR-212, miR-223, } \\
\text { miR-340, miR-365, miR-485, miR-505, } \\
\text { miR-520d, miR-629, miR-638, miR- } \\
\text { 939, miR-940, miR-1225, miR-1238 } \\
\downarrow \text { let-7i, miR-16, miR-17, miR-18a, } \\
\text { miR-18b, miR-20a, miR-20b, miR-22, } \\
\text { miR-93, miR- 96, miR-106a, miR-107, } \\
\text { miR-126, miR-130a, miR-130b, miR- } \\
\text { 151, miR-185, miR-194, miR-363, } \\
\text { miR-660 }\end{array}$ & & Soro & Exercício em cicloergômetro, ciclismo & 67 \\
\hline $\begin{array}{l}\uparrow \text { miR-7, miR-15a, miR-21, miR-26b, } \\
\text { miR-132, miR-140, miR-181a, miR- } \\
\text { 181b, miR-181c, miR-338, miR-363, } \\
\text { miR-939, miR-940, miR-1225 } \\
\downarrow \text { let-7e, miR-23b, miR-31, miR-99a, } \\
\text { miR-125a, miR-125b, miR-126, miR- } \\
\text { 130a, miR-145, miR-151, miR-199a, } \\
\text { miR-199b, miR-221, miR-320, miR- } \\
\text { 451, miR-486, miR-584, miR-652 }\end{array}$ & & PBMC & Exercício em cicloergômetro, ciclismo & 68 \\
\hline $\begin{array}{l}\uparrow \text { let-7f, miR-21, miR-29c, miR-223 } \\
\downarrow \text { let-7f, miR-21, miR-29c, miR-223 }\end{array}$ & & PBMC & Exercício de corrida & 69 \\
\hline $\begin{array}{l}\uparrow \text { miR-7, miR-29a, miR-29b, miR-29c, } \\
\text { miR-30e, miR-142, miR-192, miR-338, } \\
\text { miR-363, miR-590 } \\
\downarrow \text { let-7e, miR-126, miR-130a, miR-151, } \\
\text { miR-199a, miR-221, miR-223, miR- } \\
\text { 326, miR-328, miR-652 }\end{array}$ & & PBMC & Exercício em cicloergômetro, ciclismo & 70 \\
\hline $\begin{array}{l}\uparrow \text { miR-15a, miR-29b, miR-29c, miR- } \\
\text { 30e, miR-140, miR-324, miR-338, } \\
\text { miR-362, miR-532, miR-660 } \\
\downarrow \text { miR-23b, miR-130a, miR-151, miR- } \\
\text { 199a, miR-221 }\end{array}$ & & Soro & Exercício em cicloergômetro, ciclismo & 71 \\
\hline$\uparrow \mathrm{miR}-1, \mathrm{miR}-486, \mathrm{miR}-494$ & & Soro & $\begin{array}{l}\text { (Atletas de resistência, corredores, ciclistas e } \\
\text { triatletas) } \\
\text { Teste de exercício cardiopulmonar }\end{array}$ & 72 \\
\hline $\begin{array}{l}\uparrow \text { miR-21, miR-146a, miR-221, miR- } \\
222 \\
\uparrow \text { miR-20a, miR-21, miR-146a, miR- } \\
221 \text {, miR-222 }\end{array}$ & & Soro & $\begin{array}{l}\text { Treinamento por remada, } 5 \mathrm{~km}, 1-3 \mathrm{~h} \text { por } \\
\text { sessão, } 20-24 \text { remadas } / \mathrm{min})\end{array}$ & 73 \\
\hline $\begin{array}{l}\uparrow \text { miR-376a } \\
\downarrow \text { miR-16, miR-27a, miR-28 }\end{array}$ & & Plasma & $\begin{array}{l}\text { Treinamento de exercício aeróbico - corrida } \\
\text { (4 dias/semana) }\end{array}$ & 74 \\
\hline $\begin{array}{l}\uparrow \text { miR-19a, miR-19b, miR-20a, miR- } \\
\text { 26b, miR-143, miR-195 }\end{array}$ & $\begin{array}{l}\mathrm{p}-\mathrm{AKT} \\
\mathrm{p}-\mathrm{S} 6 \mathrm{~K} 1\end{array}$ & Soro & Exercício de resistência & 75 \\
\hline$\uparrow \mathrm{miR}-222$ & HIPK1 & Plasma & Teste ergométrico em bicicleta & 41 \\
\hline $\begin{array}{l}\uparrow \operatorname{miR}-221 \\
\downarrow \text { miR-208b, miR-221, } \\
\text { miR-21, miR-146a, } \\
\text { miR-210 }\end{array}$ & & Soro & Exercício de basquete & 76 \\
\hline
\end{tabular}


Outro estudo, com um protocolo de TEF semelhante, também evidenciou uma melhoria da capacidade funcional, com melhoria da fração de ejeção e resistência respiratória, melhoria da pressão diastólica no ventrículo esquerdo, e da qualidade de vida de pacientes chagásicos após 8 meses de treinamento. ${ }^{30}$

Um programa de reabilitação cardíaca composto do mesmo protocolo de TEF mencionado acima, com TR e alongamentos, orientação nutricional adicional e aconselhamento farmacológico para pacientes com DC, demonstrou aumento da capacidade funcional e física, melhorando a qualidade de vida de pacientes chagásicos. ${ }^{31}$

Em outro estudo importante, os pesquisadores realizaram TEF três vezes por semana em pacientes chagásicos. Eles demonstraram que o grupo que realizou exercícios aumentou o consumo de oxigênio de pico durante o exercício e a ventilação máxima por minuto, melhorando a capacidade funcional desses pacientes. ${ }^{32}$

Entretanto, apesar de demonstrar que o TEF tem efeitos benéficos para pacientes com DC, é difícil analisar os efeitos desse tipo de treinamento no nível do tecido, celular e molecular, considerando que esses estudos foram realizados em seres humanos, para quem seriam necessárias biópsias. Portanto, para investigar os possíveis mecanismos associados a esses efeitos benéficos do TEF na DC, alguns estudos foram realizados em modelos experimentais de DC in vivo.

Camundongos Balb/c realizaram TEF em uma esteira antes de serem infectados com T. Cruzi. Observou-se que o TEF reduziu o pico da parasitemia, concluindo que o TEF pode promover alterações benéficas no sistema imune e obter melhores respostas a infecções. ${ }^{33}$

Em outros estudos, foram relatados os mesmos achados que os de estudos anteriores; entretanto, eles também observaram que ratos que fizeram o treinamento obtiveram maior proteção da atividade metabólica de $\mathrm{NADH}$ em neurônios mioentéricos e maior síntese de TNF- $\alpha$ e TGF- $\beta .^{34}$ Isso contribuiu para a sobrevivência e/ou proteção de 10,3\% dos neurônios mioentéricos e sua produção imunorreativa de sintase neuronal de óxido nítrico. O grupo em treinamento, inclusive, obteve maior expressão de TNF- $\alpha$ durante a fase aguda da infecção por T. Cruzi, oferecendo benefícios ao sistema imune para preservar os neurônios nitrérgicos. ${ }^{35}$

Nesse contexto, em outro estudo, pesquisadores observaram que o grupo de TEF obteve maior expressão de TNF- $\alpha$, IFN $\gamma$, IL-6 e as quimiocinas MCP-1 e CX3CL1 durante a infecção aguda, além de alcançarem melhor capacidade física, aumento do limiar anaeróbio, aumento da atividade da catalase e do superóxido dismutase, e redução da oxidação lipídica e proteica no tecido cardíaco, demonstrando que o TEF pode ser uma estratégia interessante para aumentar a eficiência de mecanismos antioxidantes endógenos, reduzindo os danos oxidativos nesses animais. ${ }^{36}$

Outro estudo demonstrou que o TEF antes da infecção em ratos Wistar aumentou o tempo até que se atingisse a fadiga e o limiar anaeróbio, reduziu a expressão de TNF- $\alpha$, CCL-2, MCP-1 e CX3CL1, bem como a oxidação lipídica e proteica, e aumentou a expressão de IL-10, catalase e superóxido dismutase, indicando que o TEF induz um fenótipo protetor, aumentando as defesas do hospedeiro contra o agente parasítico, inclusive atenuando o processo de remodelação patológica associado à miosite musculoesquelética. ${ }^{37}$

Finalmente, em outro estudo, ratos suíços foram infectados pelo T. Cruzi após TEF de intensidade moderada em uma esteira, realizado durante 9 semanas. Os pesquisadores identificaram que o TEF conseguiu reduzir a parasitemia latente dos animais infectados submetidos a treinamento, corroborando os achados de estudos anteriores. Eles chegaram a obter menor produção de citocinas pró-inflamatórias (TNF- $\alpha$, INF $\gamma$, IL-12) e proteína quimiotática de monócitos 1 (MCP-1) durante os primeiros dias de infecção. ${ }^{38}$

Portanto, sugere-se que o TEF tenha um potencial terapêutico para a prevenção e o tratamento complementar de DC e CCC pela modulação do sistema imune. Entretanto, estudos clínicos carecem de análises morfométricas, celulares e moleculares especialmente pela análise de miRNAs para melhor entendimento dos efeitos benéficos do TEF na sinalização celular em seres humanos com DC, enquanto estudos pré-clínicos, in vivo, demandam estudos que avaliem os efeitos de TEF com DC e CCC já instaladas e não apenas no estágio de pré-infecção.

\section{Sobreposições entre miRNAs em DC e TEF}

Além disso, realizamos uma análise utilizando o diagrama de Venn, para identificar os miRNAs que foram modulados por TEF, em estudos clínicos e pré-clínicos que podem possivelmente modular miRNAs na DC.

Houve apenas 7 miRNAs expressos em DC, 95 miRNAs expressos em estudos clínicos com TEF, e 36 miRNAs expressos em estudos pré-clínicos com TEF. É interessante notar que foram identificados 7 miRNAs que tinham modulação tanto na DC quanto em estudos clínicos com TEF, 3 miRNAs comuns modulados na DC e em estudos pré-clínicos com TEF, e, principalmente, 12 miRNAs comuns modulados na CD, estudos clínicos com TEF, e estudos pré-clínicos com TEF (Figura 1). Esses 12 miRNAs são: miR-1, miR-21, miR-26b, miR-29b, miR-133a, miR-133b, miR-139, miR-145, miR-146a, miR-208a, miR-208b, miR-222.

Entretanto, desses 12 miRNAs comuns, apenas miR-133b, miR-139 e miR-208a foram identificados com um padrão de expressão diferente na DC e TEF; todos os 3 miRNAs passam por regulação descendente na DC, e ascendente, em TEF (Figura 2).

O miR-133b controla o fator de crescimento de tecido conjuntivo $(\mathrm{CTGF})^{77}$ e pode suprimir a remodelação cardíaca; ${ }^{78}$ portanto, o TEF pode ser uma alternativa excelente para controlar a remodelação cardíaca, possivelmente pela modulação do miR-133b e a modificação da sinalização celular.

O miR-139 está associado à cardiomiopatia hipertrófica, regulando a expressão do c-Jun, um fator transcricional que liga a região promotora de alguns genes para induzir a hipertrofia cardíaca, e, portanto, a superexpressão desse miRNA reduz a expressão do c-Jun e consequentemente atenua a hipertrofia cardíaca patológica, ${ }^{79}$ que pode ser uma sinalização celular pela qual o TEF suprime a hipertrofia patológica na DC, porque o TEF também aumenta a expressão desse miRNA. ${ }^{22,59}$ 


\section{Artigo de Revisão}

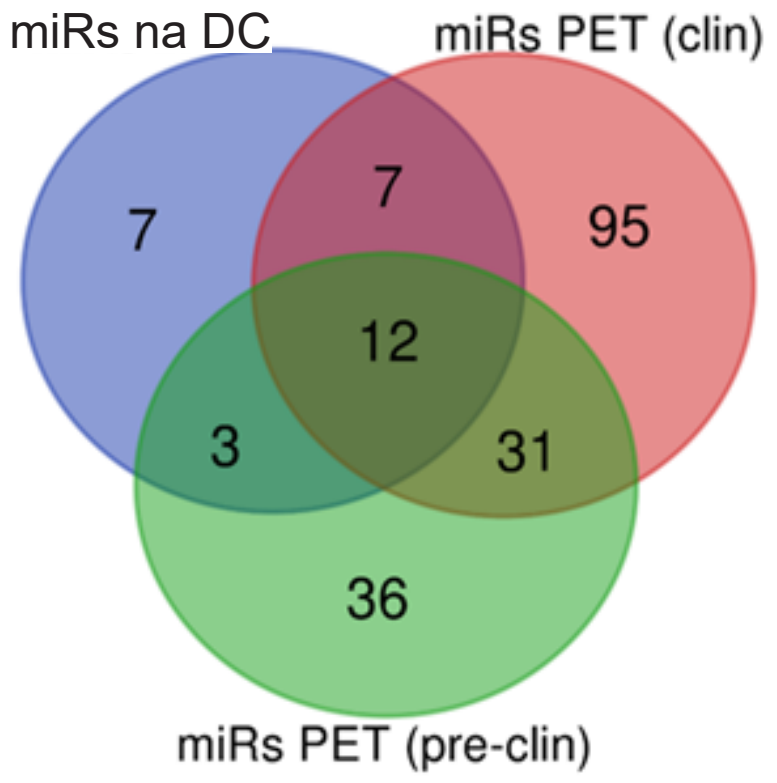

Figura 1 - 0 diagrama de Venn mostra sobreposições entre miRNAs: miRNAs (miRs) na doença de Chagas (azul), miRs TEF clin: estudos clínicos (rosa), e miRs TEF pre-clin: estudos pré-clínicos (verde).

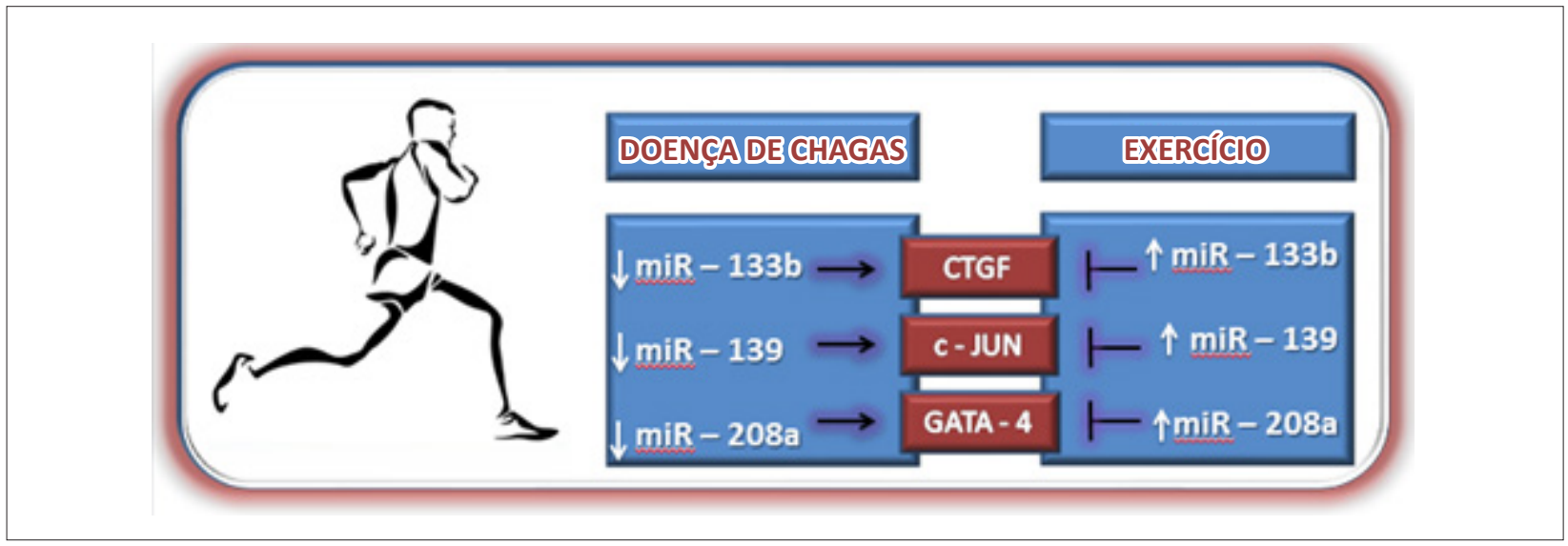

Figura 2 - miRNAs expressos na DC que podem provavelmente ser modulados por TEF.

Nesse contexto, o miR-208a regula a expressão de alguns fatores transcricionais, tais como GATA-4, que está associado à ativação de genes cardíacos pró-hipertróficos. ${ }^{80}$ Na DC, esse miRNA sofre regulação descendente, ${ }^{16}$ enquanto a TEF pode aumentar sua expressão, ${ }^{21,42}$ demonstrando, portanto, que possivelmente pode ser um mecanismo molecular pelo qual o TEF atenua a hipertrofia cardíaca nessa doença.

\section{Conclusões}

Os miRNAs participam de vários processos na patogênese da DC. Muitas evidências mostram os efeitos benéficos do TEF na DC; entretanto, ainda não há artigos na literatura que demonstrem as alterações nos mecanismos moleculares dos miRNAs que o TEF induz na DC. Dessa forma, são necessários estudos posteriores para elucidar esses mecanismos.

\section{Agradecimentos}

Este estudo foi financiado parcialmente pela Coordenação de Aperfeiçoamento de Pessoal de Nível Superior - Brasil (CAPES) - Código Financeiro 001. Agradecemos ao programa de pós-graduação em Medicina e Saúde, Faculdade de Medicina, Universidade Federal da Bahia. Gostaríamos de agradecer a Mirela Correia Improta Caria pela ajuda na criação da Figura 2. 


\section{Contribuição dos autores}

Concepção e desenho da pesquisa, Obtenção de dados e Redação do manuscrito: Improta-Caria AC; Análise e interpretação dos dados e Revisão crítica do manuscrito quanto ao conteúdo intelectual importante: Improta-Caria AC, Aras Júnior R.

\section{Potencial conflito de interesse}

Não há conflito com o presente artigo

\section{Referências}

1. Bestetti RB, Cardinalli-Neto A. Sudden cardiac death in Chagas' heart disease in the contemporary era. Int J Cardiol. 2008;131(1):9-17. doi: 10.1016/j. ijcard.2008.05.024

2. Oliveira AC, Peixoto JR, Arruda LB, Campos MA, Gazzinelli RT, Golenbock DT, et al. Expression of functional TLR4 confers proinflammatory responsiveness to Trypanosoma cruzi glycoinositolphospholipids and higher resistance to infection with T. cruzi. J Immunol. 2004;173(9):5688-96. doi: 10.4049/jimmunol.173.9.5688.

3. Nogueira LG, Santos RH, Fiorelli AI, Mairena EC, Benvenuti LA, Bocchi EA, et al. Myocardial gene expression of T-bet, GATA-3, Ror-yt, FoxP3, and hallmark cytokines in chronic Chagas disease cardiomyopathy: an essentially unopposed TH1-type response. Mediators Inflamm. 2014;2014:914326. doi: $10.1155 / 2014 / 914326$.

4. Petersen CA, Burleigh BA. Role for interleukin-1 beta in Trypanosoma cruziinduced cardiomyocyte hypertrophy. Infect Immun. 2003;71(8):4441-7. doi: 10.1128/IAI.71.8.4441-4447.2003.

5. Petersen CA, Krumholz KA, Burleigh BA. Toll-like receptor 2 regulates interleukin-1 beta-dependent cardiomyocyte hypertrophy triggered by Trypanosoma cruzi. Infect Immun. 2005;73(10):6974-80. doi: 10.1128/ IAI.73.10.6974-6980.2005.

6. Cunha Neto E, Dzau VJ, Allen PD, Stamatiou D, Benvenutti L, Higuchi ML, et al. Cardiac gene expression profiling provides evidence for cytokinopathy as a molecular mechanism in Chagas' disease cardiomyopathy. Am J Pathol. 2005;167(2):305-13. doi: 10.1016/S0002-9440(10)62976-8.

7. Rossi MA. The pattern of myocardial fibrosis in chronic Chagas' heart disease. Int J Cardiol. 1991;30(3):335-40. doi: 10.1016/0167-5273(91)90012-e.

8. Soares MB, Lima RS, Souza BS, Vasconcelos JF, Rocha LL, Santos RR, et al. Reversion of gene expression alterations in hearts of mice with chronic chagasic cardiomyopathy after transplantation of bone marrow cells. Cell Cycle. 2011;10(9):1448-55. doi: 10.4161/cc.10.9.15487.

9. Bocchi EA, Bestetti RB, Scanavacca MI, Cunha Neto E, Issa VS. Chronic Chagas Heart Disease Management: From Etiology to Cardiomyopathy Treatment. J Am Coll Cardiol. 2017;70(12):1510-1524. doi: 10.1016/j. jacc.2017.08.004.

10. Ha M, Kim VN. Regulation of microRNA biogenesis. Nat Rev Mol Cell Biol. 2014;15(8):509-24. doi: 10.1038/nrm3838.

11. Ambros V. The functions of animal microRNAs. Nature. 2004;431(7006):3505. doi: 10.1038/nature02871.

12. Krol J, Loedige I, Filipowicz W. The widespread regulation of microRNA biogenesis, function and decay. Nat Rev Genet. 2010;11(9):597-610. doi: $10.1038 /$ nrg2843.

13. Caria ACI, Nonaka CKV, Pereira CS, Soares MBP, Macambira SG, Souza BSF. Exercise Training-Induced Changes in MicroRNAs: Beneficial Regulatory Effects in Hypertension, Type 2 Diabetes, and Obesity. Int J Mol Sci. 2018;19(11):3608. doi: 10.3390/ijms19113608.

\section{Fontes de financiamento}

O presente estudo foi parcialmente financiado pela CAPES.

\section{Vinculação acadêmica}

Não há vinculação deste estudo a programas de pós-graduação.

14. Navarro IC, Ferreira FM, Nakaya HI, Baron MA, Vilar-Pereira G, Pereira IR et al. MicroRNA Transcriptome Profiling in Heart of Trypanosoma cruziInfected Mice: Parasitological and Cardiological Outcomes. PLoS Negl Trop Dis. 2015;9(6):e0003828. doi: 10.1371/journal.pntd.0003828.

15. Ferreira LRP, Ferreira FM, Laugier L, Cabantous S, Navarro IC, Cândido DS, et al. Integration of miRNA and gene expression profiles suggest a role for miRNAs in the pathobiological processes of acute Trypanosoma cruzi infection. Sci Rep. 2017;7(1):17990. doi: 10.1038/s41598-017-18080-9.

16. Ferreira LR, Frade AF, Santos RH, Teixeira PC, Baron MA, Navarro IC, et al. MicroRNAs miR-1, miR-133a, miR-133b, miR-208a and miR-208b are dysregulated in Chronic Chagas disease Cardiomyopathy. Int J Cardiol. 2014;175(3):409-17. doi: 10.1016/j.ijcard.2014.05.019.

17. Linhares-Lacerda L, Granato A, Gomes-Neto JF, Conde L, Freire-de-Lima L, Freitas EO, et al. Circulating Plasma MicroRNA-208a as Potential Biomarker of Chronic Indeterminate Phase of Chagas Disease. Front Microbiol. 2018:9:269. doi: 10.3389/fmicb.2018.00269.

18. Nonaka CKV, Macêdo CT, Cavalcante BRR, Alcântara AC, Silva DN, Bezerra MDR, et al. Circulating miRNAs as Potential Biomarkers Associated with Cardiac Remodeling and Fibrosis in Chagas Disease Cardiomyopathy. Int J Mol Sci. 2019;20(16):4064. doi: 10.3390/ijms20164064.

19. Monteiro CJ, Mota SL, Diniz LF, Bahia MT, Moraes KC. Mir-190b negatively contributes to the Trypanosoma cruzi-infected cell survival by repressing PTEN protein expression. Mem Inst Oswaldo Cruz. 2015;110(8):996-1002. doi: 10.1590/0074-02760150184.

20. Ma Z, Qi J, Meng S, Wen B, ZhangJ. Swimming exercise training-induced left ventricular hypertrophy involves microRNAs and synergistic regulation of the PI3K/AKT/mTOR signaling pathway. Eur J Appl Physiol. 2013;113(10):247386. doi: 10.1007/s00421-013-2685-9.

21. Baggish AL, Park J, Min PK, Isaacs S, Parker BA, Thompson PD, et al. Rapid upregulation and clearance of distinct circulating microRNAs after prolonged aerobic exercise. J Appl Physiol (1985). 2014;116(5):522-31. doi: 10.1152/ japplphysiol.01141.2013.

22. Souza RW, Fernandez GJ, Cunha JP, Piedade WP, Soares LC, Souza PA, et al Regulation of cardiac microRNAs induced by aerobic exercise training during heart failure. Am J Physiol Heart Circ Physiol. 2015;309(10):H1629-41. doi: 10.1152/ajpheart.00941.2014.

23. Melo SF, Barauna VG, Carneiro MA Jr, Bozi LH, Drummond LR, Natali AJ et al. Resistance training regulates cardiac function through modulation of miRNA-214. Int J Mol Sci. 2015;16(4):6855-67. doi: 10.3390/ijms16046855.

24. Rowlands DS, Page RA, Sukala WR, Giri M, Ghimbovschi SD, Hayat I, et al. Multi-omic integrated networks connect DNA methylation and miRNA with skeletal muscle plasticity to chronic exercise in Type 2 diabetic obesity. Physiol Genomics. 2014;46(20):747-65. doi: 10.1152/ physiolgenomics.00024.2014.

25. Liu SX, Zheng F, Xie KL, Xie MR, Jiang LJ, Cai Y. Exercise Reduces Insulin Resistance in Type 2 Diabetes Mellitus via Mediating the IncRNA MALAT1/ MicroRNA-382-3p/Resistin Axis. Mol Ther Nucleic Acids. 2019;18:34-44. doi: 10.1016/j.omtn.2019.08.002. 
26. Gomes JL, Fernandes T, Soci UP, Silveira AC, Barretti DL, Negrão CE, et al. Obesity Downregulates MicroRNA-126 Inducing Capillary Rarefaction in Skeletal Muscle: Effects of Aerobic Exercise Training. Oxid Med Cell Longev. 2017; 2017:2415246. doi: 10.1155/2017/2415246.

27. Melo SF, Barauna VG, Neves VJ, Fernandes T, Lara LS, Mazzotti DR, et al. Exercise training restores the cardiac microRNA- 1 and -214 levels regulating $\mathrm{Ca} 2+$ handling after myocardial infarction. BMC Cardiovasc Disord. 2015;15:166. doi: 10.1186/s12872-015-0156-4.

28. Lima MM, Rocha MO, Nunes MC, Sousa L, Costa HS, Alencar MC, et al. A randomized trial of the effects of exercise training in Chagas cardiomyopathy. Eur J Heart Fail. 2010;12(8):866-73. doi: 10.1093/eurjhf/hfq123.

29. Fialho PH, Tura BR, Sousa AS, Oliveira CR, Soares CC, Oliveira JR, et al. Effects of an exercise program on the functional capacity of patients with chronic Chagas' heart disease, evaluated by cardiopulmonary testing. Rev Soc Bras Med Trop. 2012;45(2):220-4. doi: 10.1590/s003786822012000200016 .

30. Mediano MF, Mendes FS, Pinto VL, Silva GM, Silva PS, Carneiro FM, et al. Cardiac rehabilitation program in patients with Chagas heart failure: a single-arm pilot study. Rev Soc Bras Med Trop. 2016;49(3):319-28. doi: 10.1590/0037-8682-0083-2016.

31. Mediano MFF, Mendes FSNS, Pinto VLM, Silva PSD, Hasslocher-Moreno AM, Sousa AS. Reassessment of quality of life domains in patients with compensated Chagas heart failure after participating in a cardiac rehabilitation program. Rev Soc Bras Med Trop. 2017;50(3):404-407. doi: 10.1590/0037-8682-0429-2016.

32. Mendes FSN, Mediano MFF, Souza FCC, Silva PS, Carneiro FM, Holanda MT, et al. Effect of Physical Exercise Training in Patients With Chagas Heart Disease (from the PEACH STUDY). Am J Cardiol. 2020;125(9):1413-1420. doi: 10.1016/j.amjcard.2020.01.035.

33. Schebeleski-Soares C, Occhi-Soares RC, Franzói-de-Moraes SM, Dalálio $\mathrm{MMO}$, Almeida FN, Toledo MJo, et al. Preinfection aerobic treadmill training improves resistance against Trypanosoma cruzi infection in mice. Appl Physiol Nutr Metab. 2009;34(4):659-65. doi: 10.1139/H09-053.

34. Moreira NM, Moraes SM, Dalálio MM, Gomes ML, Sant'ana DM, Araújo SM. Moderate physical exercise protects myenteric metabolically more active neurons in mice infected with Trypanosoma cruzi. Dig Dis Sci. 2014;59(2):307-14. doi: 10.1007/s10620-013-2901-9.

35. Moreira NM, Zanoni JN, Dalálio MMO, Araújo EJA, Braga CF, Araújo SM. Physical exercise protects myenteric neurons and reduces parasitemia in Trypanosoma cruzi infection. Exp Parasitol. 2014;141:68-74. doi: 10.1016/j.exppara.2014.03.005.

36. Novaes RD, Gonçalves RV, Penitente AR, Bozi LH, Neves CA, Maldonado IR, et al. Modulation of inflammatory and oxidative status by exercise attenuates cardiac morphofunctional remodeling in experimental Chagas cardiomyopathy. Life Sci. 2016;152:210-9. doi: 10.1016/j.lfs.2016.03.053.

37. Novaes RD, Gonçalves RV, Penitente AR, Cupertino MC, Maldonado IRSC, Talvani A, et al. Parasite control and skeletal myositis in Trypanosoma cruziinfected and exercised rats. Acta Trop. 2017;170:8-15. doi: 10.1016/j. actatropica.2017.02.012.

38. Lucchetti BFC, Zanluqui NG, Raquel HA, Lovo-Martins MI, Tatakihara VLH, Belém MO, et al. Moderate Treadmill Exercise Training Improves Cardiovascular and Nitrergic Response and Resistance to Trypanosoma cruzi Infection in Mice. Front Physiol. 2017; 8:315. doi: 10.3389/ fphys.2017.00315.

39. Gu Q, Wang B, Zhang XF, Ma YP, Liu JD, Wang XZ. Contribution of renin-angiotensin system to exercise-induced attenuation of aortic remodeling and improvement of endothelial function in spontaneously hypertensive rats. Cardiovasc Pathol. 2014;23(5):298-305. doi: 10.1016/j. carpath.2014.05.006.

40. Shi J, Bei $Y$, Kong $X$, Liu $X$, Lei Z, Xu T, et al. miR-17-3p Contributes to Exercise-Induced Cardiac Growth and Protects against Myocardial IschemiaReperfusion Injury. Theranostics. 2017;7(3):664-676. doi: 10.7150/ thno.15162.
41. Liu X, Xiao J, Zhu H, Wei X, Platt C, Damilano F, et al. miR-222 is necessary for exercise-induced cardiac growth and protects against pathological cardiac remodeling. Cell Metab. 2015;21(4):584-95. doi: 10.1016/j. cmet.2015.02.014.

42. Ramasamy S, Velmurugan G, Rajan KS, Ramprasath T, Kalpana K. MiRNAs with apoptosis regulating potential are differentially expressed in chronic exercise-induced physiologically hypertrophied hearts. PLoS One. 2015;10(3):e0121401. doi: 10.1371/journal.pone.0121401.

43. Xiao L, He H, Ma L, Da M, Cheng S, Duan Y, et al. Effects of miR-29a and miR-101a Expression on Myocardial Interstitial Collagen Generation After Aerobic Exercise in Myocardial-infarcted Rats. Arch Med Res. 2017;48(1):27-34. doi: 10.1016/j.arcmed.2017.01.006.

44. Fernandes T, Hashimoto NY, Magalhães FC, Fernandes FB, Casarini DE, Carmona AK, et al. Aerobic exercise training-induced left ventricular hypertrophy involves regulatory MicroRNAs, decreased angiotensinconverting enzyme-angiotensin ii, and synergistic regulation of angiotensinconverting enzyme 2-angiotensin (1-7). Hypertension. 2011;58(2):182-9. doi: 10.1161/HYPERTENSIONAHA.110.168252.

45. Soci UP, Fernandes T, Hashimoto NY, Mota GF, Amadeu MA, Rosa KT, et al. MicroRNAs 29 are involved in the improvement of ventricular compliance promoted by aerobic exercise training in rats. Physiol Genomics. 2011;43(11):665-73. doi: 10.1152/physiolgenomics.00145.2010.

46. Silva ND Jr, Fernandes T, Soci UP, Monteiro AW, Phillips MI, Oliveira EM Swimming training in rats increases cardiac MicroRNA-126 expression and angiogenesis. Med Sci Sports Exerc. 2012;44(8):1453-62. doi: 10.1249/ MSS.0b013e31824e8a36.

47. McPherson NO, Owens JA, Fullston T, Lane M. Preconception diet or exercise intervention in obese fathers normalizes sperm microRNA profile and metabolic syndrome in female offspring. Am J Physiol Endocrinol Metab. 2015;308(9):E805-21. doi: 10.1152/ajpendo.00013.2015.

48. Martinelli NC, Cohen CR, Santos KG, Castro MA, Biolo A, Frick L, et al. An analysis of the global expression of microRNAs in an experimental model of physiological left ventricular hypertrophy. PLoS One. 2014;9(4):e93271. doi: 10.1371/journal.pone.0093271.

49. Zhao Y, Ma Z. Swimming training affects apoptosis-related microRNAs and reduces cardiac apoptosis in mice. Gen Physiol Biophys. 2016;35(4):443450. doi: 10.4149/gpb_2016012.

50. Wang B, Zhang C, Zhang A, Cai H, Price SR, Wang XH. MicroRNA-23a and MicroRNA-27a Mimic Exercise by Ameliorating CKD-Induced Muscle Atrophy. J Am Soc Nephrol. 2017;28(9):2631-2640. doi: 10.1681/ ASN.2016111213.

51. Silveira AC, Fernandes T, Soci ÚPR, Gomes JLP, Barretti DL, Mota GGF, et al. Exercise Training Restores Cardiac MicroRNA-1 and MicroRNA29c to Nonpathological Levels in Obese Rats. Oxid Med Cell Longev. 2017;2017:1549014. doi: 10.1155/2017/1549014.

52. Uhlemann M, Möbius-Winkler S, Fikenzer S, Adam J, Redlich M, Möhlenkamp S, et al. Circulating microRNA-126 increases after different forms of endurance exercise in healthy adults. Eur J Prev Cardiol. 2014;21(4):484-91. doi: 10.1177/2047487312467902.

53. Aoi W, Ichikawa H, Mune K, Tanimura Y, Mizushima K, Naito Y, et al. Muscleenriched microRNA miR-486 decreases in circulation in response to exercise in young men. Front Physiol. 2013;4:80. doi: 10.3389/fphys.2013.00080.

54. Mooren FC, Viereck J, Krüger K, Thum T. Circulating microRNAs as potential biomarkers of aerobic exercise capacity. Am J Physiol Heart Circ Physiol. 2014;306(4):557-63. doi: 10.1152/ajpheart.00711.2013.

55. Gomes CP, Oliveira GP Jr, Madrid B, Almeida JA, Franco OL, Pereira RW. Circulating miR-1, miR-133a, and miR-206 levels are increased after a half-marathon run. Biomarkers. 2014;19(7):585-9. doi: 10.3109/1354750X.2014.952663.

56. Gonzalo-Calvo D, Dávalos A, Montero A, García-González Á, Tyshkovska I, González-Medina A, et al. Circulating inflammatory miRNA signature in response to different doses of aerobic exercise. J Appl Physiol. 2015;119(2):124-34. doi: 10.1152/japplphysiol.00077.2015. 
57. Clauss S, Wakili R, Hildebrand B, Kääb S, Hoster E, Klier I, et al. MicroRNAs as Biomarkers for Acute Atrial Remodeling in Marathon Runners (The miRathon Study--A Sub-Study of the Munich Marathon Study). PLoS One. 2016;11(2):e0148599. doi: 10.1371/journal.pone.0148599.

58. Min PK, Park J, Isaacs S, Taylor BA, Thompson PD, Troyanos C, et al. Influence of statins on distinct circulating microRNAs during prolonged aerobic exercise. J Appl Physiol. 2016;120(6):711-20. doi: 10.1152/ japplphysiol.00654.2015.

59. Nielsen S, Åkerström T, Rinnov A, Yfanti C, Scheele C, Pedersen BK, et al. The miRNA plasma signature in response to acute aerobic exercise and endurance training. PLoS One. 2014;9(2):e87308. doi: 10.1371/journal. pone.0087308.

60. Cui SF, Wang C, Yin X, Tian D, Lu QJ, Zhang CY, et al. Similar Responses of Circulating MicroRNAs to Acute High-Intensity Interval Exercise and Vigorous-Intensity Continuous Exercise. Front Physiol. 2016;7:102. doi: 10.3389/fphys.2016.00102.

61. Banzet S, Chennaoui M, Girard O, Racinais S, Drogou C, Chalabi H, et al. Changes in circulating microRNAs levels with exercise modality. J Appl Physiol. 2013;115(9):1237-44. doi: 10.1152/japplphysiol.00075.2013.

62. Sawada S, Kon M, Wada S, Ushida T, Suzuki K, Akimoto T. Profiling of circulating microRNAs after a bout of acute resistance exercise in humans. PLoS One. 2013;8(7):e70823. doi: 10.1371/journal.pone.0070823.

63. Zhang T, Birbrair A, Wang ZM, Messi ML, Marsh AP, Leng I, et al. Improved knee extensor strength with resistance training associates with muscle specific miRNAs in older adults. Exp Gerontol. 2015;62:7-13. doi: 10.1016/j.exger.2014.12.014.

64. Russell AP, Lamon S, Boon H, Wada S, Güller I, Brown EL, et al. Regulation of miRNAs in human skeletal muscle following acute endurance exercise and short-term endurance training. J Physiol. 2013;591(18):4637-53. doi: 10.1113/jphysiol.2013.255695.

65. Ogasawara R, Akimoto T, Umeno T, Sawada S, Hamaoka T, Fujita S. MicroRNA expression profiling in skeletal muscle reveals different regulatory patterns in high and low responders to resistance training. Physiol Genomics. 2016;48(4):320-4. doi: 10.1152/physiolgenomics.00124.2015.

66. Davidsen PK, Gallagher IJ, Hartman JW, Tarnopolsky MA, Dela F, Helge JW, et al. High responders to resistance exercise training demonstrate differential regulation of skeletal muscle microRNA expression. J Appl Physiol. 2011;110(2):309-17. doi: 10.1152/japplphysiol.00901.2010.

67. Radom-Aizik S, Zaldivar F Jr, Oliver S, Galassetti P, Cooper DM. Evidence for microRNA involvement in exercise-associated neutrophil gene expression changes. J Appl Physiol. 2010;109(1):252-61. doi: 10.1152/ japplphysiol.01291.2009.

68. Radom-Aizik S, Zaldivar F Jr, Leu SY, Adams GR, Oliver S, Cooper DM. Effects of exercise on microRNA expression in young males peripheral blood mononuclear cells. Clin Transl Sci. 2012;5(1):32-8. doi: 10.1111/j.17528062.2011.00384.x.
69. Dias RG, Silva MS, Duarte NE, Bolani W, Alves CR, Lemos JR Jr, et al. PBMCs express a transcriptome signature predictor of oxygen uptake responsiveness to endurance exercise training in men. Physiol Genomics. 2015;47(2):1323. doi: 10.1152/physiolgenomics.00072.2014.

70. Radom-Aizik S, Zaldivar F, Haddad F, Cooper DM. Impact of brief exercise on peripheral blood NK cell gene and microRNA expression in young adults. Appl Physiol. 2013;114(5):628-36. doi: 10.1152/japplphysiol.01341.2012.

71. Radom-Aizik S, Zaldivar FPJr, Haddad F, Cooper DM. Impact of brief exercise on circulating monocyte gene and microRNA expression: implications for atherosclerotic vascular disease. Brain Behav Immun. 2014;39:121-9. doi: 10.1016/j.bbi.2014.01.003.

72. Denham J, Prestes PR. Muscle-Enriched MicroRNAs Isolated from Whole Blood Are Regulated by Exercise and Are Potential Biomarkers of Cardiorespiratory Fitness. Front Genet. 2016;7:196. doi: 10.3389/ fgene.2016.00196

73. Baggish AL, Hale A, Weiner RB, Lewis GD, Systrom D, Wang F, et al. Dynamic regulation of circulating microRNA during acute exhaustive exercise and sustained aerobic exercise training. J Physiol. 2011;589(16):3983-94. doi: 10.1113/jphysiol.2011.213363.

74. Zhang T, Brinkley TE, Liu K, Feng X, Marsh AP, Kritchevsky S, et al. Circulating MiRNAs as biomarkers of gait speed responses to aerobic exercise training in obese older adults. Aging. 2017;9(3):900-913. doi: 10.18632/ aging.101199.

75. Margolis LM, Rivas DA, Berrone M, Ezzyat Y, Young AJ, McClung JP, et al Prolonged Calorie Restriction Downregulates Skeletal Muscle mTORC1 Signaling Independent of Dietary Protein Intake and Associated microRNA Expression. Front Physiol. 2016;7:445. doi: 10.3389/fphys.2016.00445.

76. Li Y, Yao M, Zhou Q, Cheng Y, Che L, Xu J, et al. Dynamic Regulation of Circulating microRNAs During Acute Exercise and Long-Term Exercise Training in Basketball Athletes. Front Physiol. 2018;9:282. doi: 10.3389/ fphys.2018.00282.

77. Gjymishka A, Pi L, Oh SH, Jorgensen M, Liu C, Protopapadakis Y, et al. miR133b Regulation of Connective Tissue Growth Factor: A Novel Mechanism in Liver Pathology. Am J Pathol. 2016;186(5):1092-102. doi: 10.1016/j. ajpath.2015.12.022.

78. Li N, Zhou H, Tang Q. miR-133: A Suppressor of Cardiac Remodeling? Front Pharmacol. 2018;9:903. doi: 10.3389/fphar.2018.00903.

79. Ming S, Shui-Yun W, Wei Q, Jian-Hui L, Ru-Tai H, Lei S, et al. miR-139$5 \mathrm{p}$ inhibits isoproterenol-induced cardiac hypertrophy by targetting c-Jun. Biosci Rep. 2018;38(2):BSR20171430. doi: 10.1042/BSR20171430.

80. Callis TE, Pandya K, Seok HY, Tang RH, Tatsuguchi M, Huang ZP, et al. MicroRNA-208a is a regulator of cardiac hypertrophy and conduction in mice. J Clin Invest. 2009;119(9):2772-86. doi: 10.1172/JCI36154. 